\title{
Left temporal craniotomy and direct microcatheterization of the middle meningeal artery for treatment of a complex temporal dural arteriovenous fistula
}

\author{
Daniel M. S. Raper, MBBS, Nasser Mohammed, MD, M. Yashar S. Kalani, MD, PhD, \\ and Min S. Park, MD \\ Department of Neurosurgery, University of Virginia Health System, Charlottesville, Virginia
}

\begin{abstract}
The preferred method for treating complex dural arteriovenous fistulae of the transverse and sigmoid sinuses is via endovascular, transarterial embolization using liquid embolysate. However, this treatment approach mandates access to distal dural feeding arteries that can be technically challenging by standard endovascular approaches. This video describes a left temporal craniotomy for direct stick microcatheterization of an endovascularly inaccessible distal posterior division of the middle meningeal artery for embolization of a complex left temporal dural arteriovenous fistula. The case was performed in the hybrid operative suite with biplane intraoperative angiography. Technical considerations, operative nuances, and outcomes are reviewed.
\end{abstract}

The video can be found here: https://youtu.be/Dnd4yHgaKcQ.

KEYWORDS dural arteriovenous fistula; endovascular; Onyx; surgical technique; transarterial embolization; video 